\title{
NUMERICAL BOUNDS FOR THE DISTRIBUTIONS OF THE MAXIMA OF SOME ONE- AND TWO-PARAMETER GAUSSIAN PROCESSES
}

\author{
CÉCILE MERCADIER, * Université Paul Sabatier
}

\begin{abstract}
We consider the class of real-valued stochastic processes indexed on a compact subset of $\mathbb{R}$ or $\mathbb{R}^{2}$ with almost surely absolutely continuous sample paths. We obtain an implicit formula for the distributions of their maxima. The main result is the derivation of numerical bounds that turn out to be very accurate, in the Gaussian case, for levels that are not large. We also present the first explicit upper bound for the distribution tail of the maximum in the two-dimensional Gaussian framework. Numerical comparisons are performed with known tools such as the Rice upper bound and expansions based on the Euler characteristic. We deal numerically with the determination of the persistence exponent.
\end{abstract}

Keywords: Stochastic process; random field; distribution of the maximum; absolutely continuous sample path; first passage time; WAFO toolbox

2000 Mathematics Subject Classification: Primary 60G60; 60G15

Secondary 60G70; 62G05

\section{Introduction}

Let $X$ be a real-valued random process defined on a compact subset of $\mathbb{R}$ or $\mathbb{R}^{2}$. In this paper we examine the following question: what is the distribution of its maximum?

In the one-parameter framework we will define the process $X$ on the interval $[0, T]$, where $T$ is a positive real. Let $M_{T}$ denote its maximum. The literature gives the exact distribution for some Gaussian processes constructed as functions of Brownian motion and also for five stationary processes (for an exhaustive list, see Azaïs and Wschebor (2002)). Most of these results are inferred from Markovian properties. When the process does not have such characteristics, the studies are usually handled by means of the tail behavior of the maximum. Indeed, the event $\left\{M_{T} \geq u\right\}$ is the disjoint union of $\{X(0) \geq u\}$ and $\mathscr{E}(T, u):=\left\{X(0)<u, M_{T} \geq u\right\}$. Then the work consists in including the event $\mathscr{E}(T, u)$ in a bigger one for which the computation of the probability is simpler and still instructive. The most classical bound includes $\mathscr{E}(T, u)$ in $\left\{U_{u}([0, T]) \geq 1\right\}$, which corresponds to the existence of an upcrossing by $X$ of the level $u$ in $[0, T]$. The associated probability has been researched since the early works of S. O. Rice and M. Kac. In general, we know by this method that, when the sample paths of $X$ are almost surely absolutely continuous,

$$
\mathrm{P}\left(M_{T} \geq u\right) \leq \mathrm{P}(X(0) \geq u)+\int_{0}^{T} \mathrm{E}\left(X^{\prime}(t)^{+} \mid X(t)=u\right) p_{X(t)}(u) \mathrm{d} t,
$$

Received 18 March 2005; revision received 26 September 2005.

* Current address: Equipe MODAL'X, Université Paris X - Nanterre, 200 avenue de la République, 92001 Nanterre cedex, France. Email address: cecile.mercadier@u-paris10.fr 
where, throughout the paper, $p_{V}(v)$ is used to denote the probability density function of a random vector $V$ at point $v$, and a prime denotes differentiation. It is well understood that, for a large level $u$, the so-called Rice upper bound (1) provides an accurate estimate of $\mathrm{P}\left(M_{T} \geq u\right)$. Azaïs and Wschebor (2002) gave a general formula, writing that $\mathscr{E}(T, u)$ is equal to the event $\left\{U_{u}([0, T]) \mathbf{1}_{\{X(0)<u\}} \geq 1\right\}$, where $\mathbf{1}_{\{.\}}$is the indicator function. The distribution tail of $M_{T}$ is thus expressed by means of an infinite series whose terms contain the factorial moments of this slight modification of the number of upcrossings. Unfortunately, the associated computational method is not satisfactory when, for a fixed level $u$, the parameter $T$ becomes large.

In a multidimensional framework we restrict ourselves to a real-valued Gaussian field (also called a two-parameter process) defined on a compact subset $S$ of the plane. Note that there are no exact formula or bounds for the distribution of its maximum, which we denote by $M_{S}$. However, the following methods make it possible to give an estimate of $\mathrm{P}\left(M_{S} \geq u\right)$ as $u$ tends to infinity.

(A) The generalization by Adler (1981, pp. 66-92) of the number of upcrossings with the integral geometry characteristic, which is equal to the Euler characteristic of $A(u)=$ $\{t \in S: X(t) \geq u\}$ if this set does not encounter the boundary of $S$.

(B) The use of the volume of tubes for particular unit-variance Gaussian fields, by Sun (1993) and Takemura and Kuriki (2002).

(C) The double sum method of Piterbarg (1996, pp. 97-141), which consists of dividing the parameter space into a finite union of smaller sets.

(D) The use of the number of local maxima, by Azaïs and Delmas (2002).

The books of Adler (1981) and Adler and Taylor (2005) give complete references for the results linked with these approaches. For instance, for a centered stationary Gaussian field $X$ of unit variance on $S=[0, T]^{2}$, let us set $\Sigma=\operatorname{var}\left(X^{\prime}(t)\right)$. Under some degeneracy conditions, we know from Adler (1981, Theorem 6.9.1, p. 160) that $\mathrm{P}\left(M_{[0, T]^{2}} \geq u\right)=\operatorname{EQA}(T, u)+$ $o\left(u \exp \left(-u^{2} / 2\right)\right)$, where

$$
\operatorname{EQA}(T, u)=\frac{T^{2} \operatorname{det}(\Sigma)^{1 / 2}}{(2 \pi)^{3 / 2}} u \mathrm{e}^{-u^{2} / 2}
$$

This has been improved by Piterbarg (1996, Corollary 7.1, p. 114), Azaïs and Delmas (2002), and Taylor et al. (2005). For all positive $\delta$, we have

$$
\mathrm{P}\left(M_{[0, T]^{2}} \geq u\right)=\operatorname{EQT}(T, u)+o\left(\exp \left(-u^{2}(1+\delta) / 2\right)\right),
$$

where

$$
\operatorname{EQT}(T, u)=\Psi(u)+\frac{T^{2} \operatorname{det}(\Sigma)^{1 / 2}}{(2 \pi)^{3 / 2}} u \mathrm{e}^{-u^{2} / 2}+T \frac{\Sigma_{11}+\Sigma_{22}}{2 \pi} \mathrm{e}^{-u^{2} / 2}
$$

and $\Psi$ is the tail of the standard Gaussian distribution. These results have a more general formulation than the one presented here; the model considered by Taylor et al. (2005) is the most advanced.

In numerous statistical problems involving random processes and fields the maximum plays a potentially important role. In nonidentifiable mixture models, the asymptotic distributions of the log-likelihood ratio test statistics rely on those of the maxima of the stochastic processes. Their 
knowledge enables us to construct well-defined tests for which we can evaluate the power; we refer the reader to Davies (1977) for pioneering work, Gassiat (2002), and Azaiis et al. (2006). This theory has found practical applications in many areas, including image processing and genetics. In a two-dimensional space a random field is used, for instance, to model spatial data such as the sea level or, more generally, any fluctuating surface. Results on the Euler characteristic have been used by Worsley (1995a), (1995b) to obtain information on images arising in astrophysics and medicine. In physics we encounter the problem of controlling the stability or the memory of a fluctuating structure over a long period (see, for instance, Molchan and Khokhlov (2004) and Ehrhardt et al. (2002)). Here a persistence exponent characterizes the probability that a random process remains below a specific level within a given interval of time; in other words that the maximum is bounded from above. It is important to know that such works consider a level very close or equal to the mean value of the process. Consequently, it is also necessary to have an estimation of the value $\mathrm{P}\left(M_{S} \geq u\right)$ for levels that are not large.

The goal of this paper is to improve approximations (1), (2), and (3) of the distribution of the maximum $M_{S}$. In both dimensional contexts, the idea is to introduce the first passage time or location (in a sense that we must define) of the level $u$ by $X$, and compute the associated probability by application of known analytical results. In Section 2 we present the formula giving the distributions of the maxima of absolutely continuous one-parameter processes (see Theorem 1). We prove the boundedness and continuity of the density function of the first passage time (see Proposition 1). Thus, the upper tail probability of the maximum takes a simple form, given below in (5), in the case of continuously differentiable Gaussian processes. For such well-behaved processes, this implicit formula can also be obtained from Rychlik (1987). As a consequence, we deduce explicit bounds for the tail probability. In Section 3 we report an estimating procedure (and case studies) that makes it possible to address some problems from the numerical point of view. To this end, we create a 'program' based on the WAFO toolbox of Brodtkorb et al. (2000) that estimates Gaussian integrals. (The WAFO toolbox is available

TABLE 1.

\begin{tabular}{|c|c|c|c|}
\hline $\begin{array}{l}\text { Bound } \\
\text { name }\end{array}$ & Description & In the text & Comment \\
\hline RICE & Rice upper bound & Equation (1) & Explicit \\
\hline UBAW & $\begin{array}{l}\text { Upper bound of Azaïs and } \\
\text { Wschebor (2002) }\end{array}$ & Section 3.3 & $\begin{array}{l}\text { See Azaïs and } \\
\text { Wschebor (2002) }\end{array}$ \\
\hline LBAW & $\begin{array}{l}\text { Lower bound of Azaiis and } \\
\text { Wschebor (2002) }\end{array}$ & Section 3.3 & $\begin{array}{l}\text { See Azaïs and } \\
\text { Wschebor (2002) }\end{array}$ \\
\hline UBPn & Upper bound for smooth processes & Equation (6) & Free value of $n$ \\
\hline UBP & Upper bound for smooth processes & Equation (6) & For $n=30$ \\
\hline LBPn & Lower bound for smooth processes & Equation (7) & Free value of $n$ \\
\hline LBP & Lower bound for smooth processes & Equation (7) & Mean for several values of $n$ \\
\hline UBNS & Upper bound for nonsmooth processes & Equation (12) & For $n=100$ \\
\hline LBNS & Lower bound for nonsmooth processes & Equation (12) & For $n=100$ \\
\hline EQA & Equivalent of Adler (1981, p. 160) & Equation (2) & See Adler (1981) \\
\hline EQT & Equivalent of Taylor et al. (2005) & Equation (3) & See Taylor et al. (2005) \\
\hline UBUS & Upper bound for 'unit-speed' fields & Equation (17) & Explicit \\
\hline LBF & Lower bound for smooth fields & Equation (18) & For $n=10$ \\
\hline UBF & Upper bound for smooth fields & Equation (19) & $\begin{array}{l}\text { For } n_{p}=100, n_{q}=15 \\
\quad \text { and } n_{r}=10\end{array}$ \\
\hline
\end{tabular}


online at http://www.maths.lth.se/matstat/wafo.) Our approximations are compared with the most classical tool, namely the Rice upper bound, and also with computations of Azaiis and Wschebor (2002). In Section 4 we provide a numerical study of two nonsmooth processes, namely Brownian motion and the Ornstein-Uhlenbeck process. In Section 5 we present an implicit formula for the distribution of a real-valued Gaussian field defined on a compact subset of the plane (see Theorem 2). In addition, we give a simple and explicit upper bound for the distribution tail in the 'unit-speed' case (see Corollary 1) that seems to be the first published bound for Gaussian fields. In a particular context, we see that this upper bound is equivalent to the EQT expansion (see Corollary 2 and the remark that follows it). In Section 6 we compare our estimates to expansions based on the integral geometry characteristic.

The paper contains several bounds, summarized in Table 1. See the relevant parts of the text for definitions of the parameters.

\section{Smooth stochastic processes}

Let $T$ denote a positive real number and consider $\{X(t), t \in[0, T]\}$ to be a real-valued stochastic process with sample paths supposed to be almost surely absolutely continuous.

Theorem 1. Let $\{X(t), t \in[0, T]\}$ be a process with almost surely absolutely continuous sample paths. We suppose that, for almost every $t$ in $[0, T], X^{\prime}(t)$ is integrable and $X(t)$ admits a density $p_{X(t)}$. Then, for every $u$ in $\mathbb{R}$,

$$
\mathrm{P}\left(M_{T} \geq u\right)=\mathrm{P}(X(0) \geq u)+\lim _{\delta \rightarrow 0} \frac{1}{\delta} \int_{u}^{u+\delta} \mathrm{d} x \int_{0}^{T} \mathrm{~d} t f(t, x),
$$

where the function $f$ is defined by $f(t, x)=\mathrm{E}\left(X^{\prime}(t)^{+} \mathbf{1}_{\{X(s)<x \forall s<t\}} \mid X(t)=x\right) p_{X(t)}(x)$.

This formula is not explicit; in particular, the supremum of the process appears in the indicator function present on the right-hand side. However, it is interesting to obtain an equality in this general framework that does not assume $X$ to be Gaussian.

Proof of Theorem 1. We write $\mathcal{E}(T, u)$ as $\left\{\tau_{u} \in(0, T)\right\}$, where $\tau_{u}$ is defined, with the convention that inf $\varnothing=-\infty$, by

$$
\tau_{u}=\inf \{t \in(0, T): X(t)=u \text { and } X(s)<u \text { for all } s<t\} .
$$

Our method is based on the indicator variable $I_{T, u}=\mathbf{1}_{\left\{\tau_{u} \in(0, T)\right\}}$, with which we can write the right-tail probability as $\mathrm{P}\left(M_{T} \geq u\right)=\mathrm{P}(X(0) \geq u)+\mathrm{E}\left(I_{T, u}\right)$. To compute the first moment of $I_{T, u}$, we consider some Borel sets, $B$ and $E$, of $\mathbb{R}$ and an absolutely continuous function $x: \mathbb{R} \rightarrow \mathbb{R}$, and we set $N(E, x, u)=\operatorname{card}\{t \in E: x(t)=u\}$. Denote by $x^{\prime}$ the derivative of $x$ in the sense of absolute continuity. If $N(E, x, u)$ is finite for almost every $u$ then we can prove, using a formula of Banach, that

$$
\int_{B} N(E, x, u) \mathrm{d} u=\int_{E}\left|x^{\prime}(t)\right| \mathbf{1}_{B}(x(t)) \mathrm{d} t .
$$

Let us define the record point set of the process as

$$
E=\{t \in[0, T]: X(s)<X(t) \text { for all } s<t\} .
$$

We have $N(E, X, u)=I_{T, u}$ and, almost surely,

$$
\int_{B} I_{T, u} \mathrm{~d} u=\int_{0}^{T}\left|X^{\prime}(t)\right| \mathbf{1}_{\{X(s)<X(t) \forall s<t\}} \mathbf{1}_{B}(X(t)) \mathrm{d} t .
$$


Taking the expectation and using Fubini's theorem, the integrability of $X^{\prime}(t)$, and the absolute continuity of $X(t)$, for every Borel set $B$ of $\mathbb{R}$ we obtain

$$
\int_{B} \mathrm{E}\left(I_{T, u}\right) \mathrm{d} u=\int_{B} \mathrm{~d} u \int_{0}^{T} \mathrm{~d} t \mathrm{E}\left(\left|X^{\prime}(t)\right| \mathbf{1}_{\{X(s)<u \forall s<t\}} \mid X(t)=u\right) p_{X(t)}(u) .
$$

Taking into account the indicator function and the conditioning, we observe that the absolute value of $X^{\prime}(t)$ is equal to $X^{\prime}(t)^{+}$and deduce that, for almost every $u$ in $\mathbb{R}$,

$$
\mathrm{E}\left(I_{T, u}\right)=\int_{0}^{T} f(t, u) \mathrm{d} t
$$

The right continuity of the function $\mathrm{P}\left(M_{T} \geq u\right)-\mathrm{P}(X(0) \geq u)$ leads to (4).

We actually again find the Rice upper bound for processes satisfying the hypotheses of Theorem 1. By defining the conditional expectation $\mathrm{E}\left(X^{\prime}(t)^{+} \mid X(t)=u\right)$ as the limit, as $\delta$ tends to 0 , of $(1 / \delta) \int_{u}^{u+\delta} \mathrm{E}\left(X^{\prime}(t)^{+} \mid X(t)=x\right) \mathrm{d} x$, we obtain

$$
\mathrm{P}\left(M_{T} \geq u\right) \leq \mathrm{P}(X(0) \geq u)+\int_{0}^{T} \mathrm{E}\left(X^{\prime}(t)^{+} \mid X(t)=u\right) p_{X(t)}(u) \mathrm{d} t .
$$

We turn now to the case of Gaussian processes. The value $f(t, u)$ is the density function at point $t$ of the passage time $\tau_{u}$, obtained by Rychlik (1987) in the case of continuously differentiable sample paths. Consequently, for such processes we can directly write

$$
\mathrm{P}\left(M_{T} \geq u\right)=\mathrm{P}(X(0) \geq u)+\mathrm{P}\left(\tau_{u} \in(0, T)\right)=\mathrm{P}(X(0) \geq u)+\int_{0}^{T} f(t, u) \mathrm{d} t .
$$

However, we propose an original proof of this formula in this framework, which seems simpler.

Proposition 1. Let $\{X(t), t \in[0, T]\}$ be a Gaussian process with almost surely continuously differentiable sample paths. We suppose that $\sigma_{T}^{2}=\min _{t \in[0, T]} \operatorname{var}(X(t))>0$. The function $\int_{0}^{T} f(t, \cdot) \mathrm{d} t$ defined in Theorem 1 is then continuous and, for every $u$ in $\mathbb{R}$,

$$
\mathrm{P}\left(M_{T} \geq u\right)=\mathrm{P}(X(0) \geq u)+\int_{0}^{T} \mathrm{E}\left(X^{\prime}(t)^{+} \mathbf{1}_{\{X(s)<u \forall s<t\}} \mid X(t)=u\right) p_{X(t)}(u) \mathrm{d} t .
$$

Consequently, the distribution of the maximum is known from only two terms. At first sight the interest of (5) could appear limited, but it lies within the upper bound (6) deduced below.

Proof of Proposition 1. Regularity of the sample paths implies that $\mathrm{E}\left(X^{\prime}(t)\right)$ and $\operatorname{var}\left(X^{\prime}(t)\right)$ are continuous, leading to the finiteness of

$$
e_{T}=\sup _{t \in[0, T]} \mathrm{E}\left(\left|X^{\prime}(t)\right|\right) \quad \text { and } \quad s_{T}^{2}=\sup _{t \in[0, T]} \operatorname{var}\left(X^{\prime}(t)\right) .
$$

Recall that if $\mathcal{N}$ is a Gaussian random variable then $\mathrm{E}\left(\mathcal{N}^{+}\right) \leq|\mathrm{E}(\mathcal{N})|+\sqrt{\operatorname{var}(\mathcal{N})}$. Applying this to $\mathcal{N}=X^{\prime}(t)$ under a conditional distribution and using the Gaussian regression formula, after simplification we obtain $f(t, u) \leq\left(e_{T}+2 s_{T}\right) / \sigma_{T}$.

Clearly, it is now sufficient to prove that $u \mapsto \mathrm{E}\left(X^{\prime}(t)^{+} \mathbf{1}_{\{X(s)<u \forall s<t\}} \mid X(t)=u\right)$ is continuous. Let $r(\cdot, \cdot)$ denote the covariance function of the process. Perform a Gaussian regression of $\left\{\left(X(s), X^{\prime}(s)\right), s<t\right\}$, with respect to the variable $X(t)$, by writing

$$
X(s)=a^{t}(s) X(t)+(t-s) U^{t}(s), \quad X^{\prime}(s)=b^{t}(s) X(t)-U^{t}(s)+(t-s) \tilde{U}^{t}(s),
$$


where, for all $s<t, a^{t}(s)=r(s, t) / r(t, t)$ and $b^{t}(s)=(\partial / \partial s) a^{t}(s)$. The processes $U^{t}$ and $\tilde{U}^{t}$ are Gaussian, nondegenerate, and independent of $X(t)$. Let us introduce $D_{t, u}=b^{t}(t) u-U^{t}(t)$ and $B_{t, u}=\mathbf{1}_{\left\{\mathcal{M}_{t, u}<0\right\}}$, where

$$
\mathcal{M}_{t, u}=\sup _{s<t}\left\{U^{t}(s)-u \frac{1-a^{t}(s)}{t-s}\right\} .
$$

We obtain $\mathrm{E}\left(X^{\prime}(t)^{+} \mathbf{1}_{\{X(s)<u \forall s<t\}} \mid X(t)=u\right)=\mathrm{E}\left(D_{t, u}^{+} B_{t, u}\right)$. For a sequence $\left(u_{n}\right)_{n}$ that converges to $u$ as $n$ tends to $\infty$, we consider

$$
\left|\mathrm{E}\left(D_{t, u_{n}}^{+} B_{t, u_{n}}\right)-\mathrm{E}\left(D_{t, u}^{+} B_{t, u}\right)\right| \leq \mathrm{E}\left(\left|D_{t, u_{n}}^{+}-D_{t, u}^{+}\right|\right)+\mathrm{E}\left(D_{t, u}^{+}\left|B_{t, u_{n}}-B_{t, u}\right|\right) .
$$

By a dominated convergence argument, the first term on the right-hand side tends to 0 . For the second term, note that

$$
\mathrm{P}\left(B_{t, u_{n}} \neq B_{t, u}\right) \leq \mathrm{P}\left(\mathcal{M}_{t, u_{n}}<0, \mathcal{M}_{t, u} \geq 0\right)+\mathrm{P}\left(\mathcal{M}_{t, u_{n}} \geq 0, \mathcal{M}_{t, u}<0\right)
$$

also tends to 0: $\mathcal{M}_{t, u_{n}} \rightarrow \mathcal{M}_{t, u}$ as $n$ tends to $\infty$ and, according to Ylvisaker (1968), $\mathcal{M}_{t, u} \neq 0$ almost surely. The integrability of $D_{t, u}^{+}$then leads to the desired conclusion.

From the theorem of Ylvisaker (1968), the hypothesis $\sigma_{T}^{2}>0$ implies that $\mathrm{P}\left(M_{T} \geq \cdot\right)$ is continuous. The proof of Proposition 1 now follows by combining this assertion with Theorem 1, and the continuity just demonstrated.

\section{Numerical applications for smooth Gaussian processes}

Let $\{X(t), t \in[0, T]\}$ be a Gaussian process satisfying the conditions of Proposition 1 and, consequently, (5). Our aim is to write upper and lower bounds for the distribution tail of the maximum of $X$. Throughout the paper we use $\varphi, \Phi$, and $\Psi$ to respectively denote the standard normal probability density function, distribution function, and tail. On the one hand, for every $n$ in $\mathbb{N}^{*}=\mathbb{N} \backslash\{0\}$, we obtain an upper bound by replacing the interval $[0, t)$ by the subdivision $\Delta_{t, n}=\{k t / n, k=0, \ldots, n-1\}$. We find that

$$
\mathrm{P}\left(M_{T} \geq u\right) \leq \mathrm{UBPn}(T, u, n, X),
$$

where

$$
\operatorname{UBPn}(T, u, n, X)=\mathrm{P}(X(0) \geq u)+\int_{0}^{T} \mathrm{E}\left(X^{\prime}(t)^{+} \mathbf{1}_{\left\{X(s)<u \forall s \in \Delta_{t, n}\right\}} \mid X(t)=u\right) p_{X(t)}(u) \mathrm{d} t .
$$

It is easy to see that (6) is valid under the assumptions of Theorem 1 if its components are continuous.

On the other hand, $\mathrm{P}\left(M_{T} \geq u\right)$ has obvious lower bounds obtained by discretization. For every $n$ in $\mathbb{N}^{*}$, we have

$$
\mathrm{P}\left(M_{T} \geq u\right) \geq \operatorname{LBPn}(T, u, n, X),
$$

where

$\operatorname{LBPn}(T, u, n, X)=1-\mathrm{P}\left(X(s) \leq u\right.$ for all $\left.s \in \tilde{\Delta}_{T, n-1}\right), \quad \tilde{\Delta}_{T, n}=\{k T / n, k=0, \ldots, n\}$.

After a description of our numerical framework, we will compare our method to the known tools discussed in the introduction. 


\subsection{MATLAB ${ }^{\circledR}$ toolbox}

For the sake of simplicity, we now restrict ourselves to centered processes with unit variance and we denote by $r$ the covariance function. Consequently, in the arguments of UBPn and LBPn, $X$ can be replaced by $r$. To illustrate our results we consider the following covariance functions.

- $r_{1}(t)=\cos (t)$, for the cosine process.

- $r_{2}(t)=\exp \left(-t^{2} / 2\right)$, the Gaussian covariance.

- $r_{3}(s, t)=\left(\mathrm{e}^{s t}-1\right) / \sqrt{\left(\mathrm{e}^{s^{2}}-1\right)\left(\mathrm{e}^{t^{2}}-1\right)}$, for the Gaussian process giving the asymptotic distribution of the likelihood ratio test in Gaussian mixtures (see Gassiat (2002) and Azaiis et al. (2006)).

- $r_{4}(t)=(\cosh (t))^{-1}$, the inverse of the hyperbolic cosine.

- $r_{5}(t)=(\sqrt{3} t)^{-1} \sin (\sqrt{3} t)$, for a low-pass process (white noise through a low-pass filter).

- $r_{6}(t)=\mathrm{e}^{-\sqrt{5}|t|}\left(\sqrt{5}|t|^{3} / 3+2 t^{2}+\sqrt{5}|t|+1\right)$, the normalization of the convolution $r_{0} * r_{0} * r_{0} * r_{0}$ of the Ornstein-Uhlenbeck covariance $r_{0}(t)=\exp (-|t|)$.

The last three covariances will only be used in Section 3.4, which is dedicated to the study of the persistence exponent. We insist on the fact that $r_{3}$, unlike the others, is nonstationary. For the stationary covariances the time has been normalized such that $\operatorname{var}\left(X^{\prime}(t)\right) \equiv 1$. This normalization, sometimes called the 'unit-speed' transformation, leads to a very simple form for the right-hand side of (1): $\Psi(u)+\varphi(u) T / \sqrt{2 \pi}$.

Numerical applications have been run under MATLAB. Our procedure is entirely based on the 'rind.m' routine from version 2.0.5 of WAFO, written by Brodtkorb et al. (2000). This routine computes Gaussian integrals that may be singular and offers several methods of integration. We made all computations with the method of the Genz (1992) algorithm, taking the option Nit $=-1$. The integration in the upper bound (6) is carried out by the MATLAB function 'quad', which approximates the integral of a function within a given error using recursive adaptive Simpson quadrature.

In theory, the larger is the parameter $n$, the better are the bounds (6) and (7): the authors of the routine advise to integrate in $\mathbb{R}^{100}$. Unfortunately, the time of estimation of the function UBPn may become too long for large values of $n$. The author thinks that the value $n=$ 30 is a satisfactory compromise. For small values of $n$ the upper tail probability is clearly underestimated by (7), whereas, when $n$ is too large in comparison with $T$, the covariance matrix associated with elements of the indicator function of (7) is almost singular. Since the true value could be overestimated for intermediate values of $T$, we have decided to make a slight randomization in its estimation. Thus,

- $\operatorname{UBPn}(T, u, n, r)$ calculates the upper bound (6) and $\operatorname{UBP}(T, u, r)=\operatorname{UBPn}(T, u, 30, r)$;

- $\operatorname{LBPn}(T, u, n, r)$ evaluates the lower bound (7) and $\operatorname{LBP}(T, u, r)$ gives the mean of $\operatorname{LBPn}(T, u, n, r)$ for $n=10,15,20, \ldots, 100$;

- $\operatorname{RICE}(T, u)$ computes the upper bound (1), which is trivial except for the covariance $r_{3}$.

These functions are available online (see Mercadier (2005)). All results are graphically presented with values interpolated by the MATLAB function 'spline'. 
TABLE 2: Estimation of $\mathrm{P}\left(M_{T} \geq 0.5\right)$ for $r_{1}$ (the cosine process).

\begin{tabular}{lcccccc}
\hline & $T=0.5$ & $T=1.5$ & $T=3.1$ & $T=4.5$ & $T=10$ & $T=15$ \\
\hline UBP & 0.3788 & 0.5192 & 0.7439 & 0.8709 & 0.8866 & 0.8840 \\
True value & 0.3788 & 0.5192 & 0.7439 & 0.8709 & 0.8825 & 0.8825 \\
LBP & 0.3786 & 0.5191 & 0.7438 & 0.8699 & 0.8797 & 0.8793 \\
\hline
\end{tabular}

TABLE 3: Estimation of $\mathrm{P}\left(M_{1} \geq u\right)$ for $r_{2}$. The 'estimates' are those of Azaïs et al. (1999, p. 112).

\begin{tabular}{lcccccc}
\hline & $u=-2$ & $u=-1$ & $u=0$ & $u=1$ & $u=2$ & $u=3$ \\
\hline UBP & 0.9944 & 0.9278 & 0.6526 & 0.2540 & 0.0442 & 0.0031 \\
Estimate & 0.9944 & 0.9279 & 0.6527 & 0.2541 & 0.0442 & 0.0031 \\
LBP & 0.9945 & 0.9281 & 0.6527 & 0.2539 & 0.0430 & 0.0025 \\
\hline
\end{tabular}

\subsection{Checking the program and evaluating the error}

On the theoretical side, the cosine process (corresponding to the covariance $r_{1}$ ) is the only smooth process from our list for which the distribution of the maximum $M_{T}$ is known. Indeed, from Berman (1971) and Delmas (2003), for any positive $u$ we have

$$
\mathrm{P}\left(M_{T} \geq u\right)= \begin{cases}\Psi(u)+\varphi(u) \frac{T}{\sqrt{2 \pi}}, & 0<T<\pi, \\ \Psi(u)+\varphi(u) \frac{T}{\sqrt{2 \pi}}-\int_{\pi}^{T} \exp \left(-\frac{u^{2}(1-\cos (t))}{\sin (t)^{2}}\right) \frac{\mathrm{d} t}{2 \pi}, & \pi \leq T<2 \pi, \\ \exp \left(-\frac{u^{2}}{2}\right), & T \geq 2 \pi .\end{cases}
$$

Because of the particular form of $\mathrm{P}\left(M_{T} \geq u\right)$, we fix a value of $u$ and study the probability as a function of the parameter $T$. We present our numerical results for the value $u=0.5$ in Table 2 .

We observe that the upper bounds obtained by the function UBP are very good for $T<\pi$. This is not surprising, since the Rice upper bound is exact on this particular domain. In general, we see in each case that the program error is reasonably small. However, this process is very special since it is periodic. In Table 3, we compare the values calculated for our second covariance function with those in another work, which is less particular.

The computation of (6) and (7) by rind.m clearly is not exact (see the case $u=-1$ in Table 3). To illustrate our best results we restrict the parameters to $T \leq 25$ and $u \geq 1$. The author thinks that on this domain we can trust the result up to an error of $10^{-3}$.

\subsection{How numerically significant is the Rice bound?}

The preceding studies show that we have a new method to estimate the value $\mathrm{P}\left(M_{T} \geq u\right)$ in some cases. However, is it a significant improvement on the classical Rice upper bound (1)? In Figure 1, we present evaluations of the function $T \mapsto \mathrm{P}\left(M_{T} \geq 1\right)$ for two different covariances.

We will say that an upper bound is numerically significant if its absolute and relative errors are respectively bounded from above by $10^{-2}$ and $10^{-1}$. When the true value is unknown we compute these errors with respect to the mean of the functions LBP and UBP. In Table 4 we 

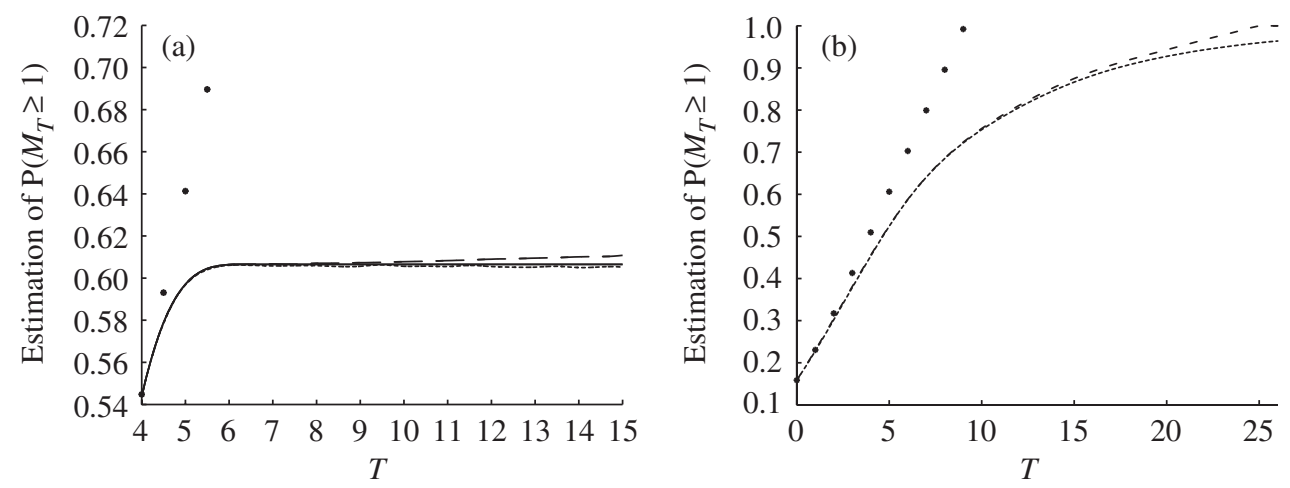

Figure 1: (a) Estimation of $\mathrm{P}\left(M_{T} \geq 1\right)$ for $r_{1}$. From top to bottom we plot RICE (points), UBP (dashed line), the true values (solid line), and LBP (dotted line). (b) Estimation of $\mathrm{P}\left(M_{T} \geq 1\right)$ for $r_{3}$. From top to bottom we plot RICE (points), UBP (dashed line), and LBP (dotted line).

TABLE 4: The maximal size of $T$ for which the given bound remains numerically significant.

\begin{tabular}{ccccccccccc}
\hline & \multicolumn{2}{c}{$u=1$} & \multicolumn{2}{c}{$u=1.5$} & \multicolumn{2}{c}{$u=2$} & \multicolumn{2}{c}{$u=2.5$} & \multicolumn{2}{c}{$u=3$} \\
\hline & RICE & UBP & RICE & UBP & RICE & UBP & RICE & UBP & RICE & UBP \\
\hline$r_{1}$ & 4 & 20 & 4 & 20 & 5 & $>25$ & 6 & $>25$ & 6 & $>25$ \\
$r_{2}$ & 2 & 20 & 3 & 20 & 8 & 20 & 19 & $>25$ & $>25$ & $>25$ \\
$r_{3}$ & 1 & 20 & 3 & 20 & 7 & 20 & 17 & $>25$ & $>25$ & $>25$ \\
\hline
\end{tabular}

estimate, for given values of $u$ and $r$, the maximal size of $T$ leading to a significant Rice upper bound and UBP bound. This demonstrates the efficiency of UBP in comparison with the Rice upper bound, particularly for intermediate and small values of the level $u$.

In this paper we propose not only an upper bound but in fact a complete estimation method: the pair (LBP, UBP). Let us compare our method to that of Azaiis and Wschebor (2002), whose complexity is close to ours. Indeed, their upper bound (denoted by UBAW) is constructed from the first three terms of the Rice series, and their lower bound (denoted by LBAW) from the first two terms. For instance, take $u=1$ and consider the covariance $r_{2}$. As can be seen from Figure 2, our method clearly becomes more efficient when $T$ is greater than 10 .

\subsection{Estimation of the persistence exponent}

Here we restrict our attention to the behavior of $-(1 / T) \log \mathrm{P}\left(M_{T}<u\right)$ as $T$ tends to $\infty$ and $u$ is fixed. This problem is mainly dealt with in the physics literature. According to Slepian (1962) and Li and Shao (2004), we know that if $X$ is an almost surely continuous centered stationary Gaussian process with positive covariance $r$, then, for every $u$ in $\mathbb{R}$, this limit exists:

$$
q(u):=\lim _{T \rightarrow \infty}\left(-\frac{1}{T}\right) \log \mathrm{P}\left(M_{T}<u\right)=\inf _{T>0}\left(-\frac{1}{T}\right) \log \mathrm{P}\left(M_{T}<u\right) .
$$

Let us consider a process $\{X(t), t \in[0, T]\}$ satisfying such hypotheses. Since the determination of $q(u)$ remains an open problem, our goal is to describe a method leading to a lower and an upper bound for it, i.e. $q_{\mathrm{LB}}(u)$ and $q_{\mathrm{UB}}(u)$ such that $q_{\mathrm{LB}}(u) \leq q(u) \leq q_{\mathrm{UB}}(u)$. Taking into 


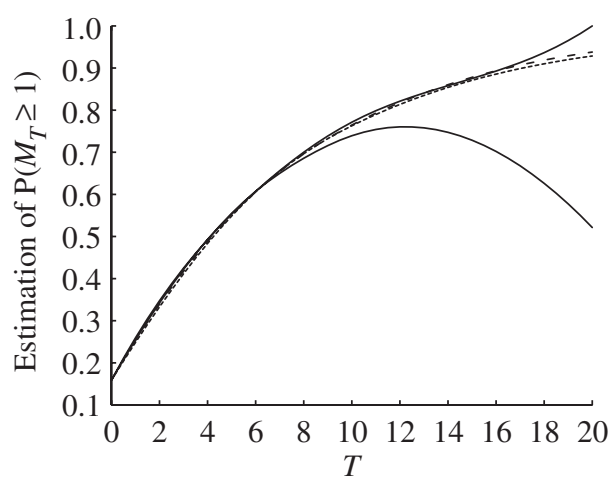

Figure 2: Estimation of $\mathrm{P}\left(M_{T} \geq 1\right)$ for the covariance $r_{2}$. From top to bottom we plot UBAW, UBP (dashed line), LBP (dotted line), and LBAW (the first and last taken from Azaïs and Wschebor (2002, p. 342)).

account the value when $T=0$, a general but reasonable approximation of the probability distribution function is given by

$$
\mathrm{P}\left(M_{T}<u\right) \sim \Phi(u) \exp (-q(u) T) .
$$

This formula explains why the term 'persistence exponent' is used for $q(u)$. We will work with the alternative definition

$$
q(u)=\lim _{T \rightarrow \infty}\left(-\frac{1}{T}\right) \log \frac{\mathrm{P}\left(M_{T}<u\right)}{\Phi(u)} .
$$

Note that now $q(u)$ is not equal to the corresponding lower bound. The function LBPn gives the following very user-friendly and efficient lower bound:

$$
q_{\mathrm{LB}}(u)=\lim _{T \rightarrow \infty}\left(-\frac{1}{T}\right) \log \frac{1-\operatorname{LBPn}(T, u, n, r)}{\Phi(u)} .
$$

Let us start with the most difficult case, in which $u=0$. For the covariance $r_{4}, \mathrm{Li}$ and Shao (2002) established that $b:=2 q(0)$ lies in $(0.5,1]$. Simulations of Dembo et al. (2002) suggest that $b=0.76 \pm 0.03$. Let us consider some covariances from the list given in Section 3.1. In practice, we observe that the limit value (9) is achieved for $T \geq 10$ and $n \geq 40$. Since $T$ is quite large, we restrict ourselves to values of $n$ greater than 40. Consequently, the limit is computed as the mean value for $T \in\{10,15,20\}$ and $n \in\{40,41, \ldots, 99,100\}$. Figure 3 displays the details of our results for the covariance $r_{4}$.

Let us summarize in Table 5 our lower bound estimates for each covariance (including $r_{5}$, which does not satisfy the positivity hypothesis of Li and Shao (2004)). Note that our conclusions for the covariance $r_{4}$ are in accordance with theoretical and numerical results previously cited. We observe that the persistence exponent $q(0)$ clearly depends on characteristics other than the local properties since, for all considered processes, we have $r(t)=1-t^{2}+O\left(t^{4}\right)$ at $t=0$. Owing to the representation with the lower bound, $q(u)$ is bounded from above by

$$
\begin{gathered}
q_{\mathrm{UB}}(u)=\inf _{T>0}\left(-\frac{1}{T}\right) \log (1-\operatorname{UBPn}(T, u, n, r)), \\
q_{\mathrm{RICE}}(u)=\inf _{T>0}\left(-\frac{1}{T}\right) \log (1-\operatorname{RICE}(T, u, r)) .
\end{gathered}
$$




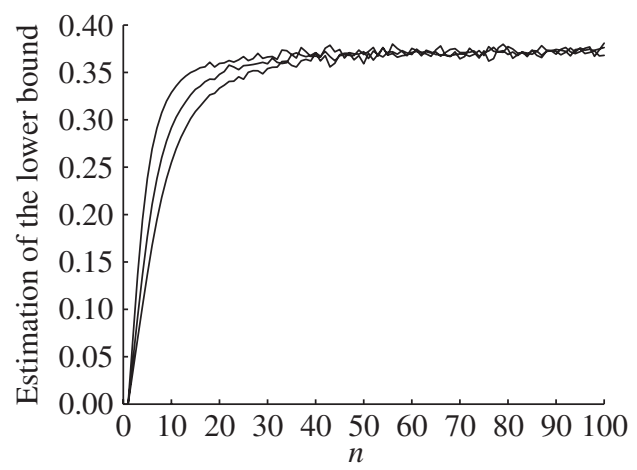

FIGURE 3: Convergence study to obtain an estimate of (9) for the covariance $r_{4}$, which represents $-(1 / T) \log (1-\operatorname{LBPn}(T, 0, n))$ as a function of $n$, for $T=10,15,20$.

TABLE 5: Lower bound of the persistence exponent $q(0)$ for our covariances.

\begin{tabular}{ccccc}
\hline & $r_{2}$ & $r_{4}$ & $r_{5}$ & $r_{6}$ \\
\hline$q_{\mathrm{LB}}(0)$ & 0.4143 & 0.3704 & 0.4521 & 0.3879 \\
\hline
\end{tabular}

TABLE 6: Estimation of persistence exponents $q(1)$ and $q(2)$ for our covariances.

\begin{tabular}{lcccc}
\hline & $r_{2}$ & $r_{4}$ & $r_{5}$ & $r_{6}$ \\
\hline$q_{\mathrm{UB}}(1)$ & 0.1445 & 0.1367 & 0.1504 & 0.1416 \\
$q_{\text {RICE }}(1)$ & 0.1960 & 0.1960 & 0.1960 & 0.1960 \\
$q_{\mathrm{LB}}(1)$ & 0.1265 & 0.1173 & 0.1308 & 0.1211 \\
$q_{\mathrm{LB}}(2)$ & 0.0222 & 0.0213 & 0.0223 & 0.0217 \\
$q_{\mathrm{UB}}(2)$ & 0.0244 & 0.0237 & 0.0244 & 0.0240 \\
$q_{\text {RICE }}(2)$ & 0.0271 & 0.0271 & 0.0271 & 0.0271 \\
\hline
\end{tabular}

Since our procedure UBPn is unstable for the value $u=0$, we cannot derive an upper bound for $q(0)$. However, $q_{\mathrm{RICE}}(0)=0.8525$, which is less instructive than the theoretical bound of 0.5 , known only in the case of $r_{4}$.

We now deal with the case in which $u$ differs from 0 , and in fact equals 1 or 2 . As we can see in Table 6 , it seems that the persistence exponent $q(u)$ becomes virtually identical for every process.

Here is a heuristic property of this behavior: for small values of $T$, it is known that

$$
\mathrm{P}\left(M_{T}<u\right) \sim \Phi(u)-T \frac{\exp \left(-u^{2} / 2\right)}{2 \pi} .
$$

The only function of the type given in (8) that has the Taylor expansion given by (10) is obtained from the substitution of $q(u)$ by

$$
h(u)=\frac{\exp \left(-u^{2} / 2\right)}{2 \pi \Phi(u)} .
$$

While the estimates in Table 6 were calculated for large values of $T$, we note that they are, for $u=2$, close to this heuristic equivalent: $h(2)=0.0220$. 


\section{Numerical implementation of nonsmooth Gaussian processes}

Let us consider in this section a centered Gaussian process $X$ whose sample paths are continuous but not differentiable. We again wish to know the distribution of its maximum. Obviously, we cannot apply (5) in order to derive upper bounds for the tail probability. Two preliminary approaches have been examined.

(M1) Use of the upper bound (6) for smoothed equivalents of $X$.

(M2) Use of the upper bound (6) with $X^{\prime}(t)$ approximated by $(X(t)-X(s)) /(t-s)$ for some $s$.

Method (M2) is theoretically justified by the result of Durbin (1985). However, both methods lead to numerical procedures that are very unstable. Consequently, since LBPn is very accurate, in this case we propose to set up an estimation method based on discretization.

We begin by describing the general ideas. Let $\{X(t), t \in[0, T]\}$ be a centered Gaussian process and let $\left\{t_{1}, \ldots, t_{n}\right\}$ be a subdivision of $(0, T)$. We assume that the interval $[0, T]$ is the disjoint union of the intervals $\left[t_{k}-d, t_{k}+d\right]$, for a positive real number $d$. We have the lower bound

$$
\mathrm{P}\left(M_{T} \geq u\right) \geq 1-\mathrm{P}\left(X\left(t_{k}\right)<u \text { for all } k=1, \ldots, n\right) .
$$

Now suppose that we know the local behavior of the process $X$ in the neighborhood of a fixed point. More precisely, assume that there exists a deterministic function $\mathcal{L}$, increasing in $[0, d]$, such that for all $t^{*}$ we have

$$
\limsup _{t \rightarrow t^{*}} \frac{\left|X(t)-X\left(t^{*}\right)\right|}{\mathcal{L}\left(\left|t-t^{*}\right|\right)}=1 \quad \text { almost surely. }
$$

We can then construct a heuristic upper bound for the distribution tail of $M_{T}$ by applying the last property to the discretization points. Define $M_{[a, b]}$ as the random variable $\sup _{t \in[a, b]} X(t)$. By compactness, each maximum is attained at a point $t_{k}^{*}$ within $\left[t_{k}-d, t_{k}+d\right]$ and, by nonrigorous use of the supremum limit, for each value of $k$ we obtain

$$
M_{\left[t_{k}-d, t_{k}+d\right]}-X\left(t_{k}\right) \lesssim \mathcal{L}(d) .
$$

In consequence, we propose the following 'upper bound':

$$
\begin{aligned}
\mathrm{P}\left(M_{T} \geq u\right) & =1-\mathrm{P}\left(M_{T}<u\right) \\
& =1-\mathrm{P}\left(M_{\left[t_{k}-d, t_{k}+d\right]}<u \text { for all } k=1, \ldots, n\right) \\
& \lesssim 1-\mathrm{P}\left(X\left(t_{k}\right)<u-\mathcal{L}(d) \text { for all } k=1, \ldots, n\right) .
\end{aligned}
$$

For numerical implementations within the nonsmooth framework, we choose the advised limit value of rind.m that computes Gaussian integrals in spaces contained in $\mathbb{R}^{100}$. Consequently, we define the MATLAB functions $\operatorname{LBNS}(T, u, r)$ and $\operatorname{UBNS}(T, u, r)$ that compute (11) and (12) by calling $\operatorname{LBPn}(T, u, 100, r)$ and $\operatorname{LBPn}(T, u-\mathcal{L}(d), 100, r)$, respectively. They are also available online (see Mercadier (2005)).

In the stationary case we consider the Ornstein-Uhlenbeck process, whose covariance function was introduced as $r_{0}(t)=\exp (-|t|)$. Locally, the behavior of such a diffusion process is the same as that of Brownian motion. Both satisfy the law of the iterated logarithm, and for every positive $\tau$ we have

$$
\mathcal{L}(\tau)=\sqrt{2 \tau \log |\log (1 / \tau)|}
$$



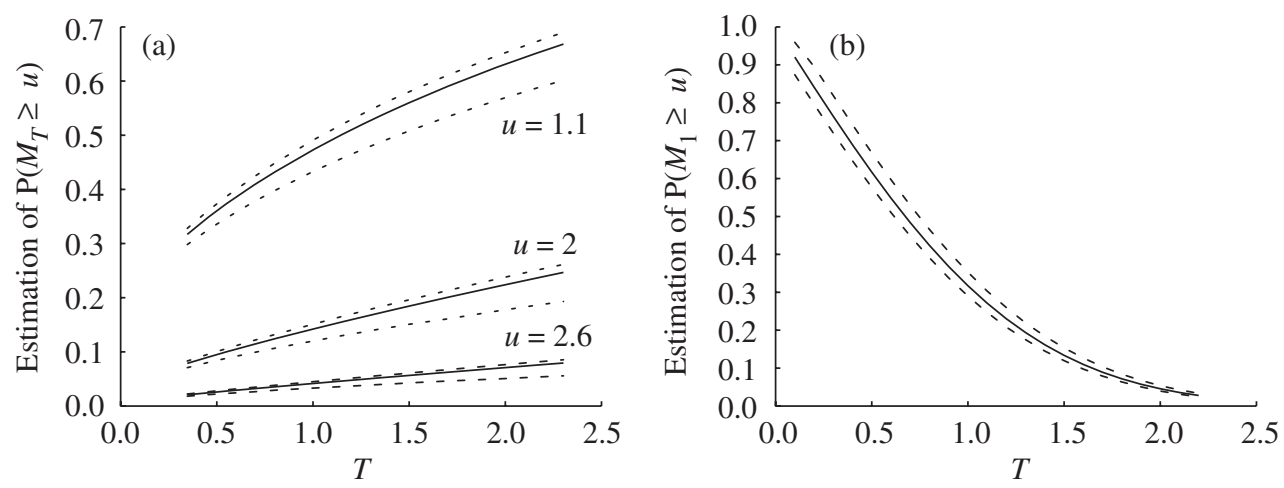

FiguRE 4: (a) Estimation of $\mathrm{P}\left(M_{T} \geq u\right)$ for $r_{0}$ and three values of $u$. For each $u$, from top to bottom we plot UBNS, the true values (DeLong (1981)), and LBNS. (b) Estimation of $\mathrm{P}\left(M_{1} \geq u\right.$ ) for $r_{7}$. From top to bottom we plot UBNS, the true values (from the reflection principle), and LBNS.

This function is increasing in $[0, d]$ if $d \leq 0.17$. For every value of $k$ we take $t_{k}=k T / n$ and $d=T / 2 n$. In this way we cover the domain $[T / 2 n, T+T / 2 n]$, which by stationarity is the same as $[0, T]$. DeLong (1981) gave the values of

$$
\mathrm{P}\left(\sup _{1<t<T} \frac{B(t)}{\sqrt{t}} \geq u\right),
$$

where $B(t)$ is the Brownian motion. We derive those of $\mathrm{P}\left(M_{T} \geq u\right)$ for the covariance $r_{0}$, using the transformation

$$
X(t)=\frac{B(\exp (2 t))}{\sqrt{\exp (2 t)}} .
$$

Figure 4(a) displays our results for three different values of $u$.

For the nonstationary case, we study the Brownian motion with covariance function $r_{7}(s, t)=$ $\min (s, t)$. As above, the function $\mathcal{L}$ is given by the law of the iterated logarithm. We make a slight modification of the subdivision in comparison with the stationary case, and cover the interval $[T / 2 n, T]$ with the union

$$
\bigcup_{k=1}^{n-1}[k T / n \pm T / 2 n] \cup[T-T / 4 n \pm T / 4 n] .
$$

Since $M_{[0, T / 2 n]}$ is negligible for large $n$, we approximate $M_{T}$ by $M_{[T / 2 n, T]}$. We present our estimations for $T=1$ in Figure 4(b).

We therefore conclude that the local behavior of a process is an interesting tool for constructing approximations of the distribution of its global maximum, in particular in the stationary case. Moreover, we note that this method leads to correct bounds even in the nonstationary case.

\section{Two-parameter Gaussian processes}

Our aim in this section is to obtain the distribution of the maximum of a two-parameter Gaussian process. We present a formula, made up of few terms, that enables us to compute 
some numerical bounds. For a subset $S$ of the plane, we denote its border and its interior by $\partial S$ and $\dot{S}$, respectively. We restrict our study to particular subsets $S$ of the plane that satisfy the following hypotheses (collectively referred to as $(\mathrm{H})$ ).

(H1) $S$ is compact.

(H2) $S$ is simply connected.

(H3) There exist a positive real number $L$ and a function $\rho:[0, L] \rightarrow \partial S$ such that $\rho$ is a $\mathcal{C}^{1}$ diffeomorphism except at a finite number of points, where it is just continuous.

In general, we could assume that $L=1$. However, the correspondence between our next result and its application to $S=[0, T]^{2}$ in Section 6 is clearer if we do not. Note that the extremal points of $[0, L]$ are identified. Moreover, we assume without loss of generality that the parameterization $\rho$ satisfies $\left\|\rho^{\prime}(\theta)\right\|_{2}=1$ for every $\theta$ in $[0, L]$.

We introduce the following notation. Denote by $\pi_{1}$ and $\pi_{2}$ the orthogonal projectors on the $x$-axis and the $y$-axis, respectively. When $t$ is a point of $S$ we sometimes write $t_{i}$ for $\pi_{i}(t)$. For $s$ and $t$ in $S$ and for all positive integers $i, j, k$, and $l$, we let

$$
X_{i, j}(t)=\frac{\partial^{i+j}}{\partial t_{1}^{i} \partial t_{2}^{j}} X(t) .
$$

The Gaussian process $Y$ is the restriction of $X$ to $\partial S$ defined by $Y(\theta)=X \circ \rho(\theta)$, and we denote by $Z$ the Gaussian field $\left(X, X_{1,0}\right)$. Recall that $M_{S}=\sup _{t \in S} X(t)$.

The approach is a generalization of the method described in Section 2. Let us choose a privileged direction in the plane: we arbitrarily choose the $y$-axis. As in the one-dimensional case, the point $t=0$ is viewed as the starting point of the interval $[0, T]$. We do the same here, assuming without loss of generality that $\pi_{2}(\rho(0))=\inf \pi_{2}(S)$, and taking $\rho(0)$ as the starting point of the subset $S$. For the one-parameter process we investigated the event $\left\{X(0)<u, M_{T} \geq u\right\}$ by studying the smallest point $t$ associated with an upcrossing of the level $u$. By analogy, we will here study points with minimal $y$-coordinates within

$$
\{t \in \partial S: X(t)=u\} \quad \text { or } \quad\left\{t \in \dot{S}, X(t)=u, X_{1,0}(t)=0\right\} .
$$

We will see that the set of such points can be written

$$
\Gamma_{t}=\left\{s \in S: s_{2} \leq t_{2}\right\}
$$

Theorem 2. Let $\{X(t), t \in S\}$ be a real-valued Gaussian field defined on a subset $S$ of $\mathbb{R}^{2}$ that satisfies hypotheses $(H)$. Assume that

(A1) the sample paths of $Z=\left(X, X_{1,0}\right)$ are almost surely continuously differentiable;

(A2) for a fixed $W$ in $\mathbb{R}^{2}$, there is almost surely no point $t$ such that both $Z(t)=W$ and $\operatorname{det}\left(Z^{\prime}(t)\right)=0$;

(A3) for all $t$ in $S$, the distribution of $Z(t)$ is nondegenerate;

(A4) for a pair $(s, t)$ in $S^{2}$ with $s_{2}=t_{2}$ and $s \neq t$, the joint distribution of $Z(s)$ and $Z(t)$ is nondegenerate. 
Then, for every u in $\mathbb{R}$,

$$
\begin{aligned}
& \mathrm{P}\left(M_{S} \geq u\right) \\
& =\mathrm{P}(Y(0) \geq u)+\int_{0}^{L} \mathrm{E}\left(\left|Y^{\prime}(\theta)\right| \mathbf{1}_{\left\{X(s) \leq u \forall s \in \Gamma_{\rho(\theta)}\right\}} \mid Y(\theta)=u\right) p_{Y(\theta)}(u) \mathrm{d} \theta \\
& \quad+\int_{S} \mathrm{E}\left(X_{2,0}(t)^{-} X_{0,1}(t)^{+} \mathbf{1}_{\left\{X(s) \leq u \forall s \in \Gamma_{t}\right\}} \mid X(t)=u, X_{1,0}(t)=0\right) p_{\left(X(t), X_{1,0}(t)\right)}(u, 0) \mathrm{d} t .
\end{aligned}
$$

We refer the reader to Azaïs and Wschebor (2005) for sufficient conditions for (A2) to hold.

The distribution of the maximum of a two-parameter Gaussian field is thus implicitly known in terms of two integrals. The interest of this formula may appear limited since the maximum appears on both sides of the equality. However, we will see in Section 6 that some accurate upper bounds can be deduced.

Proof of Theorem 2. Let us begin by studying the event

$$
\text { \{there exists a } \left.t \in S \text { such that } X(t)=u \text { and } X(s) \leq u \text { for all } s \in \Gamma_{t}\right\} .
$$

Note that the following equality holds almost surely:

\{there exists a $t \in \dot{S}$ such that $X(t)=u$ and $X(s) \leq u$ for all $\left.s \in \Gamma_{t}\right\}$

$=\left\{\right.$ there exists a $t \in \dot{S}$ such that $Z(t)=(u, 0)$ and $X(s) \leq u$ for all $\left.s \in \Gamma_{t}\right\}$.

The following lemma is easy to prove using the Bulinskaya method.

Lemma 1. Let I be a subset of $\mathbb{R}^{d}$ of dimension $m$. Let $U$ be a random field defined on an open neighborhood $O$ of $I$, with values in $\mathbb{R}^{m+k}$ for some positive integer $k$. We assume that the sample paths of $U$ are almost surely continuously differentiable and that, for every point $t$ in $O$, the density $p_{U(t)}(W)$ is bounded in a neighborhood of $W \in \mathbb{R}^{m+k}$. Then

$$
\mathrm{P}(\text { there exists a } t \in I \text { such that } U(t)=W)=0 .
$$

According to assumptions (A1) and (A4), we apply this lemma with $U(s, t)=(Z(s), Z(t))$ and $I_{n}=\left\{(s, t) \in \dot{S}^{2}: s_{2}=t_{2},\|s-t\|>1 / n\right\}$. We deduce that there almost surely exists no pair $(s, t)$ in $I=\left\{(s, t) \in \dot{S}^{2}: s_{2}=t_{2}, s \neq t\right\}$ such that $Z(s)=(u, 0)$ and $Z(t)=(u, 0)$. By the same method, we find that there almost surely exists no pair $(s, t) \in \partial S^{2}, s_{2}=t_{2}, s \neq t$, such that $X(s)=u$ and $X(t)=u$. Moreover, Ylvisaker's (1968) theorem implies that there is no local maximum with value $u$ : there is no pair $(s, t) \in S^{2}, s_{2}<t_{2}$, such that $X(s)=u$, $X(t)=u$, and $X(z) \leq u$ for all $z \in \Gamma_{t}$. As a consequence,

$$
\begin{aligned}
\left\{M_{S} \geq u\right\}= & \{Y(0) \geq u\} \\
& \cup\left\{\text { there exists a } t \in S \text { such that } X(t)=u \text { and } X(s) \leq u \text { for all } s \in \Gamma_{t}\right\}
\end{aligned}
$$

almost surely, where the union is almost surely disjoint and the existence of such a point $t$ (i.e. the occurrence of the final event) implies its uniqueness. We find that

$$
\mathrm{P}\left(M_{S} \geq u\right)=\mathrm{P}(Y(0) \geq u)+P_{1}(u)+P_{2}(u),
$$


where

$$
\begin{aligned}
& P_{1}(u)=\mathrm{E}\left(\operatorname{card}\left\{t \in \partial S: X(t)=u, X(s) \leq u \text { for all } s \in \Gamma_{t}\right\}\right), \\
& P_{2}(u)=\mathrm{E}\left(\operatorname{card}\left\{t \in \dot{S}: Z(t)=(u, 0), X(s) \leq u \text { for all } s \in \Gamma_{t}\right\}\right) .
\end{aligned}
$$

We start by computing the term $P_{1}(u)$. The resulting number can be expressed by means of the function $\rho$ as

$$
\operatorname{card}\left\{\theta \in(0, L): Y(\theta)=u, X(s) \leq u \text { for all } s \in \Gamma_{\rho(\theta)}\right\} .
$$

By the same arguments as those used in the proof of Theorem 1, and with the record point set $E=\left\{\theta \in(0, L): X(s) \leq Y(\theta)\right.$ for all $\left.s \in \Gamma_{\rho(\theta)}\right\}$, for almost every $u$ in $\mathbb{R}$ we obtain

$$
P_{1}(u)=\int_{0}^{L} \mathrm{E}\left(\left|Y^{\prime}(\theta)\right| \mathbf{1}_{\left\{X(s) \leq u \forall s \in \Gamma_{\rho(\theta)}\right\}} \mid Y(\theta)=u\right) p_{Y(\theta)}(u) \mathrm{d} \theta .
$$

The main difference between the treatment of $\int_{0}^{T} f(t, \cdot) \mathrm{d} t$ in the proof of Proposition 1 and that of the right-hand side of (14) is that the process $Y$ may lose some regularity at a finite number of points. Consequently, we here use the regularity assumption (A1) to dominate this integrand, independently of $u$, by an integrable function. Then we also perform a Gaussian regression of $X(s)$ with respect to $Y(\theta)$, to deduce that this integral is a continuous function of $u$.

We now turn to the study of the term $P_{2}(u)$. We compute it using the next lemma (proved later), which is a generalization of the Rice formula result of Azaïs and Wschebor (2005).

Lemma 2. Let $Z: S \subset \mathbb{R}^{2} \rightarrow \mathbb{R}^{2}$ be a Gaussian field satisfying assumptions (A1), (A2), and (A3). Use the supremum norm on $\mathcal{C}(S, \mathbb{R})$, the space of continuous functions from $S$ to $\mathbb{R}$, and assume further that $g: \mathcal{C}(S, \mathbb{R}) \times S \rightarrow \mathbb{R}$ is continuous and bounded. Then, for every $W$ in $\mathbb{R}^{2}$,

$$
\mathrm{E}\left(\sum_{t \in \dot{S} \cap Z^{-1}(W)} g(Z, t)\right)=\int_{\dot{S}} \mathrm{E}\left(\left|\operatorname{det}\left(Z^{\prime}(t)\right)\right| g(Z, t) \mid Z(t)=W\right) p_{Z(t)}(W) \mathrm{d} t .
$$

Both sides of this equation are continuous functions of $W$.

Let $\left(k_{n}\right)_{n}$ be a sequence of continuous and monotone functions such that $k_{n}(x)=1$ if $x \leq 0$ and $k_{n}(x)=0$ if $x>1 / n$. We can verify that the limit of $k_{n}(x)$ with respect to $n$ is $\mathbf{1}_{(-\infty, 0]}(x)$. By applying Lemma 2 with $W=(u, 0)$ and $g_{n}(Z, t)=k_{n}\left(\sup _{s \in \Gamma_{t}} Z(s)-u\right)$, by monotone and dominated convergence arguments we find that

$$
\begin{aligned}
& P_{2}(u) \\
& \quad=\int_{\dot{S}} \mathrm{E}\left(\left|\operatorname{det}\left(Z^{\prime}(t)\right)\right| \mathbf{1}_{\left\{X(s) \leq u \forall s \in \Gamma_{t}\right\}} \mid Z(t)=(u, 0)\right) p_{Z(t)}(u, 0) \mathrm{d} t \\
& \quad=\int_{S} \mathrm{E}\left(X_{2,0}(t)^{-} X_{0,1}(t)^{+} \mathbf{1}_{\left\{X(s) \leq u \forall s \in \Gamma_{t}\right\}} \mid X(t)=u, X_{1,0}(t)=0\right) p_{\left(X(t), X_{1,0}(t)\right)}(u, 0) \mathrm{d} t .
\end{aligned}
$$

Both sides of these equations are continuous functions of $u$.

To summarize, we know that (13) is true for almost every real number $u$ and that its righthand side is a continuous function of $u$. We deduce the equality in full generality by application of the theorem of Ylvisaker (1968). 
Proof of Lemma 2. Let $\beta: \mathbb{R}^{2} \rightarrow \mathbb{R}$ be a continuous function with compact support and let $N_{W}^{Z}(I)$ denote the number of solutions $t$ to the equation $Z(t)=W$ in a compact subset $I$ of $\dot{S}$. By applying the co-area formula of Federer (1969, p. 243), we can prove that

$$
\int_{\mathbb{R}^{2}} \beta(W) \sum_{t \in \dot{S} \cap Z^{-1}(W)} g(Z, t) \mathrm{d} W=\int_{\dot{S}} \beta(Z(t))\left|\operatorname{det}\left(Z^{\prime}(t)\right)\right| g(Z, t) \mathrm{d} t .
$$

The main difficulty here is to dominate the number of elements in $Z^{-1}(W)$. Define for each positive real number $\eta$ the subset $I_{\eta}=\{t \in S:\|t-s\| \geq \eta$ for all $s \notin S\}$ and consider a continuous nondecreasing function $F$ with $F(x)=0$ for $x \leq \frac{1}{2}$ and $F(x)=1$ for $x \geq 1$. Let us take $\beta(W)$ as the product $\alpha_{\Delta, \eta}(W) f(W)$. Here $f$ is a continuous function with compact support and

$$
\alpha_{\Delta, \eta}(u)=F\left(\frac{1}{2 \Delta} \inf _{s \in I_{\eta}}\left[\lambda_{\min }\left(Z^{\prime}(s)\right)+\|Z(s)-W\|\right]\right)\left(1-F\left(\frac{2}{\Delta} \omega_{Z^{\prime}}(\eta)\right)\right),
$$

where $\lambda_{\min }\left(Z^{\prime}(s)\right)$ and $\omega_{Z^{\prime}}$ are respectively the smallest eigenvalue and the continuity modulus of $Z^{\prime}$. The function $\alpha_{\Delta, \eta}(W)$ is a tool for controlling the value of the number of solutions to $Z(t)=W$ in $I_{\eta}$. In this context, by taking the expectation of both sides of (15) we deduce the equality of the following two expressions for almost every $W \in \mathbb{R}^{2}$ :

$$
\begin{gathered}
\mathrm{E}\left(\alpha_{\Delta, \eta}(W) \sum_{t \in I_{\eta} \cap Z^{-1}(W)} g(Z, t)\right) \\
\int_{I_{\eta}} \mathrm{E}\left(\left|\operatorname{det}\left(Z^{\prime}(t)\right)\right| \alpha_{\Delta, \eta}(W) g(Z, t) \mid Z(t)=W\right) p_{Z(t)}(W) \mathrm{d} t .
\end{gathered}
$$

To obtain the conclusion of Lemma 2, it is then sufficient to prove that both expressions are continuous functions of $W$, and to take the limit in both $\eta$ and $\Delta$. Each step can be made following the arguments of Azaiis and Wschebor (2005, Theorem 2.1), so we omit the remainder of the proof.

From Theorem 2 we obtain an upper bound that is easily computable in the case of a 'unitspeed' Gaussian field. Since only equivalents of such tail probabilities are proposed in the literature, this is new and is one of our main results.

Corollary 1. Let $\{X(t), t \in S\}$ be a centered Gaussian field satisfying the conditions of Theorem 2. Assume, in addition, that

(A5) $\operatorname{var}(X(t))=1$;

(A6) $\operatorname{var}\left(X^{\prime}(t)\right)$ equals the identity matrix;

(A7) $\operatorname{var}\left(X_{2,0}(t)\right)$ is constant and we set $c=\left(\operatorname{var}\left(X_{2,0}\right)-1\right)^{1 / 2}$.

Then, for every $u$ in $\mathbb{R}$,

$$
\mathrm{P}\left(M_{S} \geq u\right) \leq \Psi(u)+\frac{\lambda_{1}(\partial S)}{\pi} \exp \left(-\frac{u^{2}}{2}\right)+\frac{\lambda_{2}(S)}{(2 \pi)^{3 / 2}}\left[c \varphi\left(\frac{u}{c}\right)+u \Phi\left(\frac{u}{c}\right)\right] \exp \left(-\frac{u^{2}}{2}\right),
$$

where $\lambda_{1}$ and $\lambda_{2}$ denote the Lebesgue measures on $\mathbb{R}$ and $\mathbb{R}^{2}$, respectively. 
Note that we can write a bound (depending on an integral with respect to the variable $t$ ) without assumption (A7). Unfortunately, in terms of dimension this framework seems to be the only one in which we can find an easily computable bound.

Proof of Corollary 1. In (13) the integrand associated with the boundary of $S$ reduces to

$$
\mathrm{E}\left(\left|Y^{\prime}\right|\right) p_{Y}(u)=\frac{2}{\sqrt{2 \pi}} \frac{\exp \left(-u^{2} / 2\right)}{\sqrt{2 \pi}},
$$

since, for any $\theta \in[0, L]$, we have $\mathrm{E}(Y(\theta))=\mathrm{E}\left(Y^{\prime}(\theta)\right)=0$, $\operatorname{var}(Y(\theta))=1$, and $\operatorname{var}\left(Y^{\prime}(\theta)\right)=$ $\left\|\rho^{\prime}(\theta)\right\|_{2}^{2}=1$. Note that, for any $t$ in $S$,

- $X_{2,0}(t)$ and $X_{0,1}(t)$ are independent of $X_{1,0}(t)$;

- $\operatorname{cov}\left(X_{2,0}(t), X(t)\right)=-1$;

- $X_{2,0}(t)$ and $X_{0,1}(t)$ are independent whether or not conditioned by $X(t)$; and

- $X_{0,1}(t)$ and $X(t)$ are independent.

The following is thus an upper bound of the integrand associated with the interior of $S$ :

$$
\mathrm{E}\left(X_{0,1}^{+}\right) \mathrm{E}\left(X_{2,0}^{-} \mid X=u\right) p_{\left(X, X_{1,0}\right)}(u, 0)=\frac{1}{\sqrt{2 \pi}}\left[c \varphi\left(\frac{u}{c}\right)+u \Phi\left(\frac{u}{c}\right)\right] \frac{\exp \left(-u^{2} / 2\right)}{2 \pi} .
$$

The result follows.

We now propose some improvements of the upper bound (16) in the case $S=[0, T]^{2}$. Similar improvements actually hold for every subset $S$ whose parameterization has a derivative, $\rho^{\prime}$, constant on a subinterval of $[0, L]$.

Corollary 2. Let $\left\{X(t), t \in[0, T]^{2}\right\}$ be a Gaussian field satisfying the conditions of Corollary 1 . Then, for every $u$ in $\mathbb{R}$,

$$
\mathrm{P}\left(M_{[0, T]^{2}} \geq u\right) \leq \operatorname{UBUS}(T, u)
$$

with

$$
\operatorname{UBUS}(T, u)=\Psi(u)+\frac{T}{\pi} \exp \left(-\frac{u^{2}}{2}\right)+\frac{T^{2}}{(2 \pi)^{3 / 2}}\left[c \varphi\left(\frac{u}{c}\right)+u \Phi\left(\frac{u}{c}\right)\right] \exp \left(-\frac{u^{2}}{2}\right) .
$$

Using the asymptotics of $\Phi$ we see that, as $u$ becomes large, this upper bound satisfies

$$
\operatorname{UBUS}(T, u) \sim \Psi(u)+\frac{T}{\pi} \exp \left(-\frac{u^{2}}{2}\right)+\frac{T^{2} u}{(2 \pi)^{3 / 2}} \exp \left(-\frac{u^{2}}{2}\right),
$$

which is exactly the expansion EQT. This can also be expressed by saying that

$$
0 \leq \operatorname{UBUS}(T, u)-\operatorname{EQT}(T, u) \leq \frac{T^{2}}{\sqrt{2} \pi^{2} u^{2}} \exp \left(-\frac{3}{4 u^{2}}\right)
$$

Proof of Corollary 2. The difference between (16) and (17) comes from the second term, which corresponds to the boundary of $S$. We begin by applying the theorem of Ylvisaker (1968), which states that the maximum almost surely does not take the fixed value $u$ as local extremum. 
Consequently, the second integral of (13) is reduced to an integral on $[0, T] \times\{0\} \cup\{0\} \times$ $[0, T] \cup\{T\} \times[0, T]$. The integral on $[0, T] \times\{0\}$ is bounded from above by

$$
\int_{0}^{T} \mathrm{E}\left(X_{1,0}(\theta, 0)^{+} \mid X(\theta, 0)=u\right) p_{X(\theta, 0)}(u) \mathrm{d} \theta=\frac{T}{\sqrt{2 \pi}} \varphi(u) .
$$

Taking into account the fact that $\left\{X(s) \leq u\right.$ for all $\left.s \in \Gamma_{(0, \theta)}\right\} \subset\left\{X_{1,0}(0, \theta) \leq 0\right\}$ under the condition $\{X(\theta, 0)=u\}$, the integral on $\{0\} \times[0, T]$ is bounded from above by

$$
\int_{0}^{T} \mathrm{E}\left(X_{0,1}(0, \theta)^{+} \mathbf{1}_{\left\{X_{1,0}(0, \theta) \leq 0\right\}} \mid X(0, \theta)=u\right) p_{X(0, \theta)}(u) \mathrm{d} \theta=\frac{T}{2 \sqrt{2 \pi}} \varphi(u) .
$$

Similar arguments imply that the integral on $\{T\} \times[0, T]$ is bounded from above by

$$
\int_{0}^{T} \mathrm{E}\left(X_{0,1}(T, \theta)^{+} \mathbf{1}_{\left\{X_{1,0}(T, \theta) \geq 0\right\}} \mid X(T, \theta)=u\right) p_{X(T, \theta)}(u) \mathrm{d} \theta=\frac{T}{2 \sqrt{2 \pi}} \varphi(u),
$$

and the second term of (16) can be replaced by $T \exp \left(-u^{2} / 2\right) / \pi$. This completes the proof.

\section{Numerical implementation of smooth two-parameter Gaussian processes}

In this section we numerically develop (13), which provides the distribution of the maximum of a Gaussian field defined on a two-dimensional compact set. We restrict our attention to isotropic centered Gaussian fields of unit variance defined on $S=[0, T]^{2}$. A lower bound is obtained, as in Section 3, by discretizing $S$ using the grid

$$
\nabla_{T, n}=\{(k T / n, l T / n), k=0, \ldots, n, l=0, \ldots, n\} .
$$

We have

$$
\mathrm{P}\left(M_{[0, T]^{2}} \geq u\right) \geq \operatorname{LBF}(T, u, n),
$$

where $\operatorname{LBF}(T, u, n)=1-\mathrm{P}\left(X(s)<u\right.$ for all $\left.s \in \nabla_{T, n}\right)$.

Now we deduce an upper bound discretizing $\Gamma_{t}$, which appears in the indicator functions of the conditional expectations of (13), using the subset $\Gamma_{t, n}=\Gamma_{t} \cap \nabla_{T, n}$. In the next bound, we apply the same argument as in the proof of Corollary 2 to reduce, in (13), the integration on the boundary of $[0, T]^{2}$ to one on $[0, T] \times\{0\} \cup\{0\} \times[0, T] \cup\{T\} \times[0, T]$. Since the field on the last two factors has the same properties, we obtain

$$
\mathrm{P}\left(M_{[0, T]^{2}} \geq u\right) \leq \operatorname{UBF}\left(T, u, n_{p}, n_{q}, n_{r}\right),
$$

where

$$
\operatorname{UBF}\left(T, u, n_{p}, n_{q}, n_{r}\right)=\Psi(u)+\varphi(u)\left[P_{n_{p}}(T, u)+2 Q_{n_{q}}(T, u)+R_{n_{r}}(T, u)\right]
$$

and

$$
\begin{aligned}
P_{n}(T, u)= & \int_{0}^{T} \mathrm{E}\left(X_{1,0}(\theta, 0)^{+} \mathbf{1}_{\left\{X(s) \leq u \forall s \in \Gamma_{(\theta, 0), n}\right\}} \mid X(\theta, 0)=u\right) \mathrm{d} \theta, \\
Q_{n}(T, u)= & \int_{0}^{T} \mathrm{E}\left(X_{0,1}(0, \theta)^{+} \mathbf{1}_{\left\{X(s) \leq u \forall s \in \Gamma_{(0, \theta), n}\right\}} \mid X(0, \theta)=u\right) \mathrm{d} \theta, \\
R_{n}(T, u)= & \frac{1}{\sqrt{2 \pi} \Sigma_{1,1}} \\
& \times \iint_{[0, T]^{2}} \mathrm{E}\left(X_{2,0}(t)^{-} X_{0,1}(t)^{+} \mathbf{1}_{\left\{X(s) \leq u \forall s \in \Gamma_{t, n}\right\}} \mid X(t)=u, X_{1,0}(t)=0\right) \mathrm{d} t .
\end{aligned}
$$




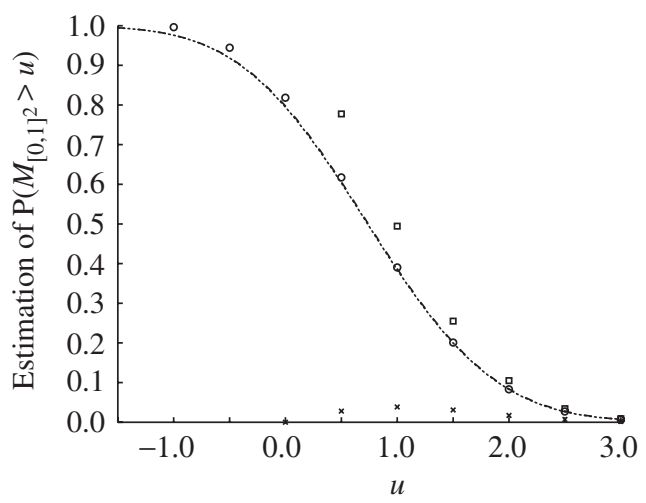

(a)

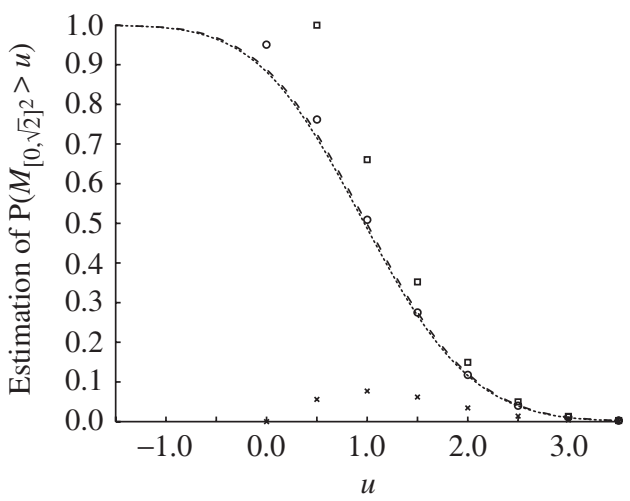

(b)

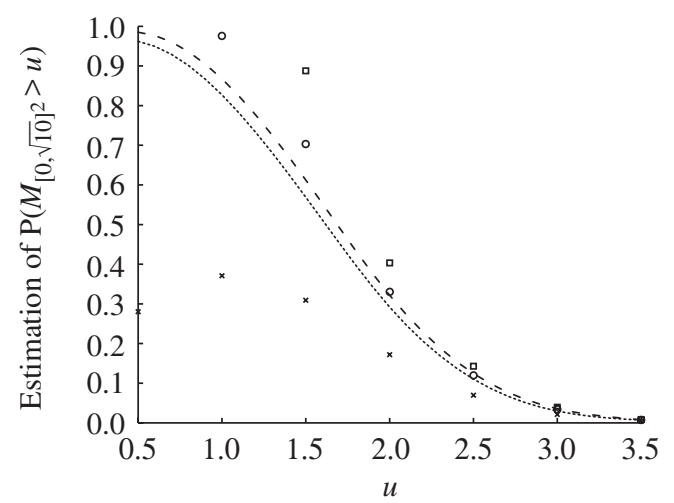

(c)

Figure 5: (a) Estimation of $\mathrm{P}\left(M_{[0, T]^{2}} \geq u\right)$ for $T^{2}=1$. (b) Estimation of $\mathrm{P}\left(M_{[0, T]^{2}} \geq u\right)$ for $T^{2}=2$. (c) Estimation of $\mathrm{P}\left(M_{[0, T]^{2}} \geq u\right)$ for $T^{2}=10$. In each case, from top to bottom we plot UBUS (squares), EQT (circles), UBF (dashed line), LBF (dotted line), and EQA (crosses).

Our goal is to compare this estimation method to the known expansions (2) and (3) presented in the introduction. To this end, we consider the Gaussian covariance function $r_{8}(s, t)=$ $\mathrm{e}^{-\|s-t\|^{2} / 2}$. In order to compute UBF and LBF we call the WAFO routine rind.m, which computes Gaussian integrals (see Section 3 for details on the WAFO toolbox). In particular,

- $\operatorname{UBF}(T, u)$ computes the upper bound (19) with $n_{p}=100, n_{q}=15$, and $n_{r}=10$;

- $\operatorname{LBF}(T, u)$ gives the value of the lower bound (18) for $n=10$.

These functions are available online (see Mercadier (2005)).

The difference between the order of $n_{p}$ and the other parameters (for instance $n_{q}$ ) comes from the fact that, for all $\theta$ in $[0, T], \Gamma_{(\theta, 0), n}$ is included in $[0, T]$. Considering the definition of $\nabla_{T, n}$, it is important to observe that when $n_{q}=15$ we consider $15^{2}$ points in the indicator function associated with $Q$.

In Figure 5 we display numerical estimates of $\mathrm{P}\left(M_{[0, T]^{2}} \geq u\right)$ for several values of $T^{2}$. The first comment concerning these numerical results is the accuracy of the expansion EQT when $T$ is small with respect to $u$. It is also interesting to note that the upper bound UBUS converges relatively quickly to the true value for intermediate levels. Consequently, such a bound could 
be of use, for instance, to properly justify the use of EQT in statistical tests. Finally, for small surfaces we are assured that our method gives the distribution of the maximum. For larger surfaces, the method remains accurate in solving problems of this complexity.

\section{Acknowledgements}

The author wishes to thank Professor I. Rychlik for his suggestions concerning the field case, and his assistance in the use of WAFO. The author also thanks Professors J.-M. Azaïs and M. Wschebor for authorization to use their data in Figure 2 and for their helpful comments.

\section{References}

AdLer, R. J. (1981). The Geometry of Random Fields. John Wiley, Chichester.

Adler, R. J. And TAYlor, J. E. (2005). Random fields and geometry. Preprint. Available at http://iew3.technion.ac.il/ $\sim$ radler/grf.pdf.

AzAïs, J.-M. AND Delmas, C. (2002). Asymptotic expansions for the distribution of the maximum of Gaussian random fields. Extremes 5, 181-212.

AzAïs, J.-M. AND Wschebor, M. (2002). The distribution of the maximum of a Gaussian process: Rice method revisited. In In and Out of Equilibrium (Mambucaba, 2000), Birkhäuser, Boston, MA, pp. 321-348.

AzAïs, J.-M. AND Wschebor, M. (2005). On the distribution of the maximum of a Gaussian field with $d$ parameters. Ann. Appl. Prob. 15, 254-278.

Azaïs, J.-M., Cierco-Ayrolles, C. and Croquette, A. (1999). Bounds and asymptotic expansions for the distribution of the maximum of a smooth stationary Gaussian process. ESAIM Prob. Statist. 3, 107-129.

Azaïs, J.-M., Gassiat, É. ANd Mercadier, C. (2006). Asymptotic distribution and power of the likelihood ratio test for mixtures: bounded and unbounded cases. To appear in Bernoulli.

Berman, S. M. (1971). Excursions above high levels for stationary Gaussian processes. Pacific J. Math. 36, 63-79.

BrodtKorb, P. A. et al. (2000). WAFO - a Matlab toolbox for analysis of random waves and loads. In Proc. 10th Internat. Offshore Polar Eng. Conf., Vol. III (Seattle, 2000), ed. J. S. Chung, ISOPE, Seattle, WA, pp. 343-350.

DAVIES, R. B. (1977). Hypothesis testing when a nuisance parameter is present only under the alternative. Biometrika 64, $247-254$.

Delmas, C. (2003). Projections on spherical cones, maximum of Gaussian fields and Rice's method. Statist. Prob. Lett. 64, 263-270.

DeLong, D. M. (1981). Crossing probabilities for a square root boundary by a Bessel process. Commun. Statist. A Theory Meth. 10, 2197-2213.

Dembo, A., Poonen, B., Shao, Q.-M. and Zeitouni, O. (2002). Random polynomials having few or no real zeros. J. Amer. Math. Soc. 15, 857-892.

Durbin, J. (1985). The first-passage density of a continuous Gaussian process to a general boundary. J. Appl. Prob. 22, 99-122.

Ehrhardt, G. C. M. A., Bray, A. J. and Majumdar, S. N. (2002). Persistence of a continuous stochastic process with discrete-time sampling: non-Markov processes. Phys. Rev. E 65, 13 pp.

FEDERER, H. (1969). Geometric Measure Theory. Springer, New York.

Gassiat, É. (2002). Likelihood ratio inequalities with applications to various mixtures. Ann. Inst. H. Poincaré Prob. Statist. 6, 897-906.

Genz, A. (1992). Numerical computation of multivariate normal probabilities. J. Comput. Graph. Statist. 1, 141-149.

LI, W. V. And Shao, Q.-M. (2002). A normal comparison inequality and its applications. Prob. Theory Relat. Fields 122, 494-508.

LI, W. V. AND Shao, Q.-M. (2004). Lower tail probabilities for Gaussian processes. Ann. Prob. 32, $216-242$.

Mercadier, C. (2005). MAGP. Maximum analysis for Gaussian processes (one and two parameters). Available at http://www.lsp.ups-tlse.fr/Fp/Mercadier/MAGP.html.

Molchan, G. And KHoKhlov, A. (2004). Small values of the maximum for the integral of fractional Brownian motion. J. Statist. Phys. 114, 923-946.

Piterbarg, V. I. (1996). Asymptotic Methods in the Theory of Gaussian Processes and Fields (Transl. Math. Monogr. 148). American Mathematical Society, Providence, RI.

Rychlik, I. (1987). A note on Durbin's formula for the first-passage density. Statist. Prob. Lett. 6, 425-428.

SlePIAn, D. (1962). The one-sided barrier problem for Gaussian noise. Bell System Tech. J. 41, 463-501.

Sun, J. (1993). Tail probabilities of the maxima of Gaussian random fields. Ann. Prob. 21, 34-71.

TAKemura, A. AND Kuriki, S. (2002). On the equivalence of the tube and Euler characteristic methods for the distribution of the maximum of Gaussian fields over piecewise smooth domains. Ann. Appl. Prob. 12, 768-796. 
Taylor, J., Takemura, A. And Adler, R. J. (2005). Validity of the expected Euler characteristic. Ann. Prob. 33, 1362-1396.

WORSLEY, K. J. (1995a). Boundary corrections for the expected Euler characteristic of excursion sets of random fields, with an application to astrophysics. Adv. Appl. Prob. 27, 943-959.

Worsley, K. J. (1995b). Estimating the number of peaks in a random field using the Hadwiger characteristic of excursion sets, with applications to medical images. Ann. Statist. 23, 640-669.

YlVISAKER, D. (1968). A note on the absence of tangencies in Gaussian sample paths. Ann. Math. Statist. 39, $261-262$. 